\title{
Emotional processing in functional neurological disorder: a review, biopsychosocial model and research agenda
}

\author{
Susannah Pick, ${ }^{1}$ Laura H Goldstein, ${ }^{2}$ David L Perez, ${ }^{3,4}$ Timothy R Nicholson ${ }^{1}$
}

\begin{abstract}
- Additional material is published online only. To view please visit the journal online (http://dx.doi.org/10.1136/ jnnp-2018-319201).

${ }^{1}$ Section of Cognitive Neuropsychiatry, Institute of Psychiatry, Psychology \& Neuroscience, King's College London, London, UK 2Department of Psychology, Institute of Psychiatry, Psychology \& Neuroscience, King's College London, London, UK

${ }^{3}$ Department of Neurology, Functional Neurology Research Group, Cognitive Behavioural Neurology Unit, Massachusetts General Hospital, Harvard Medical School, Boston, Massachusetts, USA ${ }^{4}$ Department of Psychiatry, Neuropsychiatry Unit,

Massachusetts General Hospital, Harvard Medical School, Boston, Massachusetts, USA
\end{abstract}

Correspondence to Dr Susannah Pick, Section of Cognitive Neuropsychiatry, Institute of Psychiatry, Psychology \& Neuroscience, King's College London, London SE5 8AF, UK; susannah.pick@ kcl.ac.uk

DLP and TRN contributed equally.

Received 10 July 2018 Revised 20 October 2018 Accepted 29 October 2018 Published Online First 19 November 2018

Check for updates

(C) Author(s) (or their employer(s)) 2019. No commercial re-use. See rights and permissions. Published by BMJ.

To cite: Pick S, Goldstein LH, Perez DL, et al. J Neurol Neurosurg Psychiatry 2019:90:704-711.

\section{ABSTRACT}

Functional neurological disorder (FND) is a common and highly disabling disorder, but its aetiology remains enigmatic. Conceptually, there has been reduced emphasis on the role of psychosocial stressors in recent years, with a corresponding increase in neurobiological explanations. However, a wealth of evidence supports the role of psychosocial adversities (eg, stressful life events, interpersonal difficulties) as important risk factors for FND. Therefore, there is a need to integrate psychosocial (environmental) and neurobiological factors (eg, sensorimotor and cognitive functions) in contemporary models of FND. Altered emotional processing may represent a key link between psychosocial risk factors and core features of FND. Here, we summarise and critically appraise experimental studies of emotional processing in FND using behavioural, psychophysiological and/or neuroimaging measures in conjunction with affective processing tasks. We propose that enhanced preconscious (implicit) processing of emotionally salient stimuli, associated with elevated limbic reactivity (eg, amygdala), may contribute to the initiation of basic affective/defensive responses via hypothalamic and brainstem pathways (eg, periaqueductal grey). In parallel, affect-related brain areas may simultaneously exert a disruptive influence on neurocircuits involved in voluntary motor control, awareness and emotional regulation (eg, sensorimotor, salience, central executive networks). Limbic-paralimbic disturbances in patients with FND may represent one of several neurobiological adaptations linked to early, severe and/or prolonged psychosocial adversity. This perspective integrates neurobiological and psychosocial factors in FND and proposes a research agenda, highlighting the need for replication of existing findings, multimodal sampling across emotional response domains and further examination of emotional influences on sensorimotor and cognitive functions in FND populations

\section{INTRODUCTION}

Functional neurological (conversion) disorder (FND) is defined by the presence of neurological symptoms (eg, sensorimotor, cognitive) that are not explained by identifiable neurological pathology. ${ }^{1}$ FND can present similarly to almost any neurological illness (eg, epilepsy, stroke, Parkinson's disease) and represents a notable proportion of neurology outpatient referrals. ${ }^{2}$ FND often results in severe and/or chronic symptoms with considerable impact on patients' social/occupational functioning, in addition to significant healthcare and societal costs. ${ }^{3}$ Nevertheless, there exists ongoing inconsistency in diagnostic classification and terminology for the disorder and its subtypes. ${ }^{14}$ Box 1 outlines the terminology used throughout this article.

Biopsychosocial frameworks acknowledge the variety of predisposing (eg, psychosocial adversity, gender, physical illness, exposure to symptom/ illness models), precipitating (eg, physical injury, mental health symptoms, interpersonal conflict, other stressors) and perpetuating (eg, avoidance, illness beliefs/expectations, social isolation) factors that can contribute to FND. ${ }^{36}{ }^{6}$ However, the exact mechanism(s) underlying FND symptoms are still not fully understood and there is no well-accepted explanatory model. Contemporary explanations have moved away from psychodynamic trauma-focused models and instead emphasise dysfunction of higher-order cognitive processes. ${ }^{7-9}$ Nevertheless, the importance of psychosocial adversity in FND cannot be underestimated given that rates of early-life and proximal adverse events have been repeatedly found to be higher in FND samples relative to controls. ${ }^{10}$ Stressful life-events including abuse/neglect, ongoing relationship disturbances, occupational stress and caring responsibilities are commonly reported. ${ }^{611-13}$

Patients with FND also frequently report difficulties in emotional functioning, including anxiety, depression, alexithymia and/or affect dysregulation. ${ }^{314} 15$ Greater psychosocial adversity and/or affective dysfunction are associated with poorer quality of life, greater symptom severity, reduced resilience, elevated dissociation and poorer prognosis in FND samples. ${ }^{16-25}$ Psychological interventions (eg, cognitive behavioural therapy) seeking to identify interactions between emotions, unhelpful thoughts/behaviours, psychosocial difficulties and FND symptoms show emerging efficacy in treating FND, ${ }^{26}$ underscoring an important role for altered emotional processing in this population.

There has been increasing research interest in emotional processing in FND in recent years, moving beyond self-report measures to controlled experimental task-based studies. Here, we critically review the experimental literature on emotional processing in FND to date, including only studies using behavioural, psychophysiological and/or functional neuroimaging measures. We then discuss the implications of these data for the pathophysiological basis of FND and propose an agenda for future research. 
Box 1 Terminology and abbreviations

$\mathrm{FND}=$ functional neurological disorder

FND-seiz=FND with seizures

FND-movt=FND with abnormal movements (eg, tremor, gait, dystonia)

FND-par=FND with paralysis/paresis

Additional terms for FND in the literature reviewed: conversion disorder, dissociative (neurological) disorder, psychogenic (neurological disorder)

Additional terms for FND-seiz in the literature reviewed: non-epileptic attacks/seizures, dissociative seizures/convulsions, psychogenic non-epileptic seizures

\section{EMOTIONAL PROCESSING IN FND: A NARRATIVE REVIEW}

We aimed to identify published experimental research studies on emotional processing in FND since 1965. We searched Embase, PsychInfo and Medline/PubMed databases using the following terms: conversion disorder, functional neurological, hysteria/hysterical, psychogenic, non*epileptic seizures, dissociative seizures, combined with 'emotion"' and 'affect"'. Studies involving the presentation of emotionally valenced stimuli (ie, pleasant/appetitive or unpleasant/aversive) combined with the measurement of at least one response domain (ie, subjective/ behavioural, psychophysiological, neuroimaging) within laboratory settings were included. Studies using only self-report measures and/or qualitative techniques were excluded, as were case reports/series and conference proceedings. The identified studies and key findings are summarised below and in online supplementary table 1 .

\section{Behavioural and psychophysiological findings}

Facial expression processing

Increased preconscious (implicit) attentional biases for angry and/ or happy faces have been observed in people with FND-seiz. ${ }^{27-29}$ The attentional bias for angry faces correlated positively with sexual abuse history and basal cortisol levels, ${ }^{27} 28$ whereas attentional bias for happy expressions was positively associated with seizure frequency in another study. ${ }^{29}$ Emotional facial expressions also disproportionately disrupted ongoing cognitive processing in people with FND-seiz, including working memory ${ }^{30}$ and taskswitching performance. ${ }^{31}$

There is some evidence of altered explicit (conscious, intentional) facial emotion processing in people with FND. Reduced accuracy for recognising emotional facial expressions and reduced phasic skin conductance responses (SCRs) was observed in FND-seiz participants in one study. ${ }^{15}$ Altered reaction times for explicit identification of sad and happy faces have also been reported in children and adolescents with FND, ${ }^{32}$ alongside elevated heart rate (HR) and reduced heart rate variability (HRV) in the same sample. ${ }^{33}$ Additionally, avoidant behavioural responses to angry faces have been observed in FND-seiz. ${ }^{34}$ Three studies, however, did not report differences in accuracy for facial expression identification in people with FND, suggesting the need for further research. 323536

\section{Theory of $\mathrm{mind} / \mathrm{mentalising}$}

A small number of studies indicated possible 'Theory of Mind (ToM)' or mentalising deficits in FND populations. ${ }^{3637}$ Impaired 'affective ToM' was observed in a sample with mixed-symptom $\mathrm{FND}^{37}$ and an overall pattern of 'overmentalising' has been reported in people with FND-seiz. ${ }^{36}$ The latter tendency to 'overmentalise' was positively associated with perceived stress in the previous month in that group. ${ }^{36}$

\section{Affective picture paradigms}

Altered subjective, psychophysiological, motor and/or somatosensory responses have been observed in FND samples during exposure to affective images from the International Affective Picture System ${ }^{38}$ (IAPS). ${ }^{39-43}$ Different samples or subgroups of individuals with FND displayed elevations in either subjective or somatic (autonomic, reflexive) responses to IAPS images, but not elevations in both together, ${ }^{4-43}$ suggesting diminished integration of conscious and bodily emotional experiences.

Possible interactions between emotional and somatomotor responses during processing of IAPS stimuli have also been identified. Blakemore et $a l^{39}$ for example, found evidence for facilitated manual output force in people with FND-movt while viewing negative IAPS images. Fiess et $a l^{40}$ reported increased FND symptoms and reduced discomfort threshold after intentional emotion regulation during IAPS exposure. These findings indicate a possible amplifying effect of negative emotion and/ or intentional emotion regulation on somatosensory processing.

\section{Emotional learning and memory}

There is preliminary evidence for deficits in instrumental learning in people with mixed FND symptoms, observed using a monetary reward/loss paradigm. ${ }^{44}$ However, an earlier study found that prior associative conditioning of auditory stimuli with emotional faces had no effect on subsequent performance on a subjective agency task involving the same auditory stimuli. ${ }^{45}$ Further research on associative and instrumental learning is necessary to expand on these initial findings.

There is as yet no evidence for declarative emotional memory impairment in FND samples. Brown et al ${ }^{46}$ tested memory for emotional and neutral words in people with FND-par, reporting an overall deficit on word recall that was not specific to the emotion condition. Furthermore, Aybek et $a l^{47}$ noted no between-group differences on behavioural responses for personalised life event reminders in people with FND-par.

\section{Elevated affective (autonomic) arousal}

Elevated autonomic arousal at baseline or during affective processing has been observed using varied psychophysiological measures. ${ }^{15} 27333441-43$ Findings include elevated salivary cortisol, ${ }^{34} \mathrm{HR},{ }^{33}$ skin conductance levels, ${ }^{15} \mathrm{SCRs}^{41}$ and lower $\mathrm{HRV}^{273342}$ In addition, potentiated startle responses have been observed in patients with FND-movt. ${ }^{43}$

\section{Summary — behavioural and psychophysiological findings}

FND is associated with altered social-emotional cognition, including an implicit attentional bias towards facial emotion and disrupted behavioural and autonomic responses. Baseline hyperarousal, altered autonomic responses and discordance between subjective and objective responses during affective processing have been demonstrated. Preliminary evidence suggests possible instrumental learning deficits and abnormal sensorimotor responses during affective processing tasks.

\section{Task-based neuroimaging studies}

Figure 1 summarises the neural regions in which alterations have been most consistently observed during affective processing in people with FND, along with possible functional connectivity differences. 

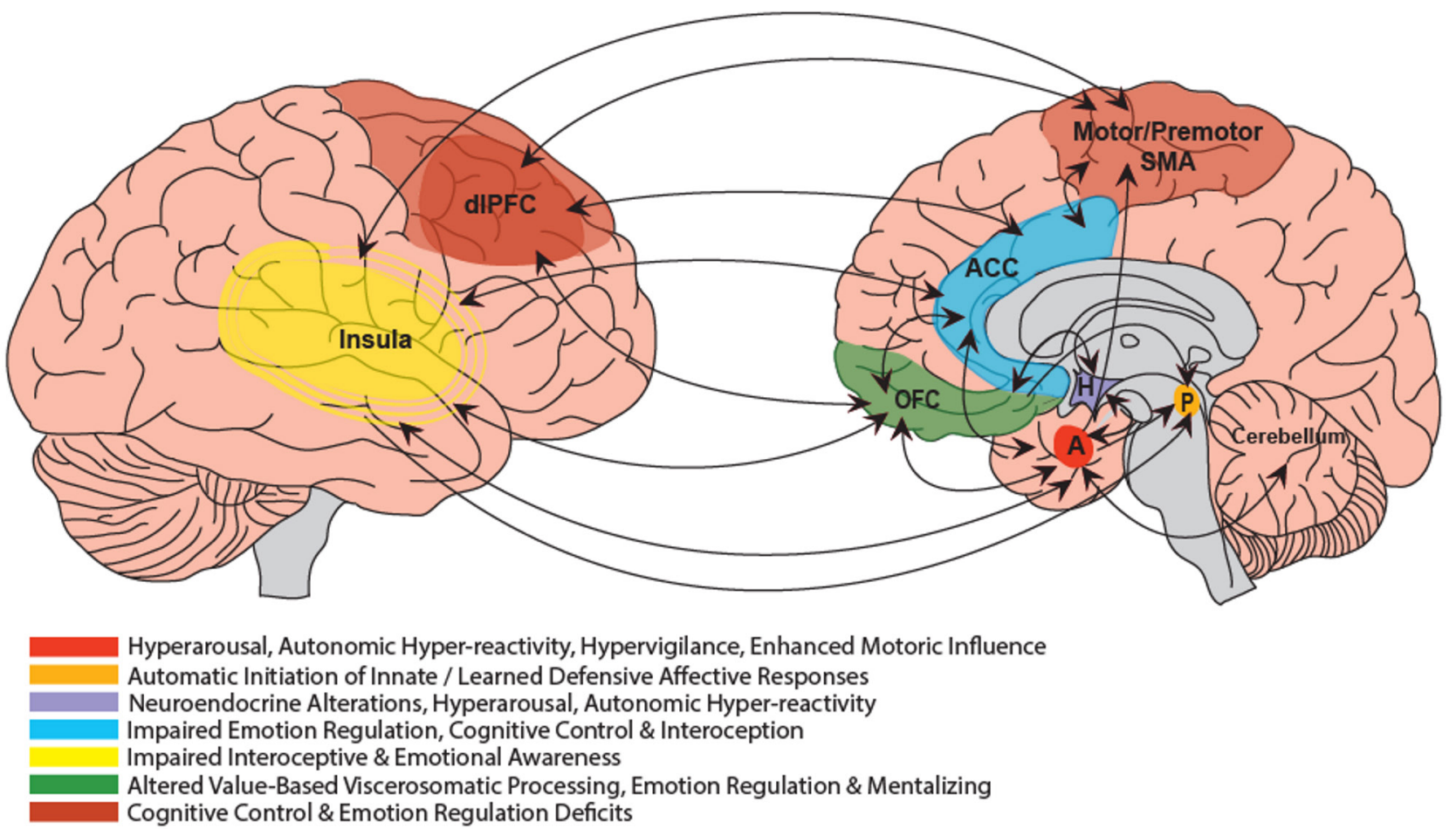

Figure 1 Schematic representation of key regional abnormalities and emerging functional connectivity alterations in FND during emotional processing. Main findings from the literature include heightened limbic (amygdalar) and motor (SMA/PMA/M1, cerebellum) activations; enhanced limbic-motor network functional connectivity; altered prefrontal and paralimbic (ACC, dIPFC, OFC, insula) engagement and elevated functional connectivity with the motor system. A, amygdala; ACC, anterior cingulate gyrus*; dIPFC, dorsolateral prefrontal cortex; $\mathrm{H}$, hypothalamus; OFC, orbitofrontal cortex*; P, periaqueductal grey; SMA, supplementary motor area. ${ }^{*}$ Both ACC and OFC project to periaqueductal grey and hypothalamus.

\section{Facial expression processing}

Altered activation has been frequently observed in affect-related regions during facial expression processing tasks. Three studies reported increased amygdalar activation for one or more emotionally valenced facial expressions. ${ }^{48-50}$ Interestingly, in one study, elevated amygdala activation was seen only during passive movement of the affected limb in patients with FND-par. ${ }^{49}$ Increased activity has also been reported in several other limbic-paralimbic regions across different FND samples, including the periaqueductal grey (PAG), dorsolateral prefrontal cortex (dlPFC), parahippocampal gyrus and cingulate cortex/ paracingulate gyrus. ${ }^{35} 5051$ However, decreased orbitofrontal cortex (OFC), insular and parahippocampal gyrus engagement was noted in patients with FND-seiz in some contrasts. ${ }^{35}$

Another pattern observed commonly during facial emotion processing is altered activity in motor regions. For example, increased activity has been reported in the supplementary motor area (SMA)/premotor cortex, ${ }^{50}$ precentral gyrus ${ }^{35}$ and cerebellum. ${ }^{52}$ Conversely, reduced activations in the precentral gyrus, putamen and cerebellum have also been noted in other contrasts. ${ }^{35} 51$ A particularly important finding is greater functional connectivity between the amygdala and motor circuits during facial emotion processing tasks. ${ }^{48} 49$

\section{Affective picture paradigms}

Alterations in limbic/paralimbic and motor circuit activity (ie, PFC, posterior cingulate, insula, amygdala, hippocampus, cerebellum, putamen) have also been reported in several studies during exposure to IAPS stimuli. ${ }^{39405253}$ Furthermore, increased amygdala-middle frontal gyrus functional connectivity has also been demonstrated. ${ }^{51}$
Emotional learning and memory

Amygdalar hyperactivation has been reported during monetary losses in people with FND, alongside a trend towards diminished dIPFC engagement. ${ }^{44}$ Patients with FND-par also exhibited increased motor, temporal and $\mathrm{dIPFC}$ activity during exposure to illness-relevant life event reminders in the study by Aybek et al. ${ }^{47}$ This same sample also displayed increased connectivity between amygdala and motor areas (SMA) during the experiment.

\section{Summary-neuroimaging findings}

The most consistent findings are increased amygdala reactivity, heightened motor circuit activation, altered prefrontal engagement and enhanced motor-limbic circuit functional connectivity during affective processing tasks across several FND subgroups.

\section{DISCUSSION}

\section{Altered social-emotional cognition}

There is considerable evidence for impaired social-emotional cognition in individuals with FND. The best supported behavioural finding is an implicit/preconscious attentional bias towards emotional facial expressions in people with FND-seiz, ${ }^{27-29}$ which is linked to adverse life events, ${ }^{27}$ hypothalamic-pituitary-adrenal (HPA-axis) dysfunction ${ }^{28}$ and symptom severity. $^{29}$ These behavioural data link directly to elevated amygdalar activity observed during implicit facial expression processing, ${ }^{48-50}$ which together suggest enhanced affective salience of these stimuli.

Increased recruitment of a subcortical 'unconscious' processing stream (eg, thalamo-amygdala pathway), ${ }^{54}$ along with initiation of basic affective responses via the hypothalamus and PAG, could 
arguably lead to this automatic and rapid attentional allocation to such stimuli. Reduced thalamic volumes ${ }^{5556}$ and elevated PAG activation ${ }^{50}$ reported in FND samples provide additional support for this suggestion. Furthermore, Perez et $a l^{57}$ noted that individual differences in amygdala and PAG volumes correlated positively with mental health symptoms, trait anxiety and role limitations due to affective disturbances in a mixed FND cohort. Together, these findings support an overlap between structural and functional alterations in the pathophysiology of FND.

There is some evidence for explicit facial expression processing differences in both children and adults with FND, linked to prior psychosocial stressors and/or maladaptive attachment styles. ${ }^{15} 32$ These behavioural findings are supported by emotion processing alterations in regions such as the amygdala, OFC and insula. ${ }^{35}{ }^{48-50}$ Differences in facial expression processing have also been noted in other somatic symptom disorders. ${ }^{58} 59$ The studies identifying impaired mentalising in FND samples ${ }^{36} 37$ further point towards difficulties in accurately inferring others' mental states and emotional experiences.

These alterations in social-emotional cognition, if replicated, may be important features in future theoretical perspectives and psychological interventions for FND.

\section{Impaired interoceptive awareness}

A discrepancy between subjective (cognitive) responses and psychophysiological measures during emotional processing was observed by several investigators, ${ }^{41-43}$ suggesting reduced integration between conscious emotional experience and somatic responses. Visceromotor responses to emotional stimuli may not be accurately perceived or interpreted by people with FND, representing a failure of 'interoception'. Another study, which examined neuroendocrine responses to social stress, also indicated discordance between subjective and neuroendocrine stress responses in patients with FND-par. ${ }^{60}$ There is also preliminary experimental evidence for interoceptive deficits on the classic heartbeat detection task in patients with FND. ${ }^{61}$

A potential neurobiological basis for interoceptive deficits in FND may involve the insula. In the studies reviewed here, the insula was less engaged during incidental processing of neutral facial expressions in people with FND-seiz ${ }^{35}$ and during unpleasant/neutral IAPS images in patients with FND-movt. ${ }^{52}$ In addition, the insula has been implicated in the broader structural and functional neuroimaging literature on FND. ${ }^{62-65}$ Furthermore, anterior insular volumes correlated negatively with symptom severity in women with mixed FND. ${ }^{2157}$

Poorer performance on measures of interoceptive accuracy has also been described in individuals with other somatoform disorders ${ }^{5966}$ and training in interoceptive accuracy can lead to reduced somatic symptoms in these patients. ${ }^{67}$ Together, these findings highlight the potential for transdiagnostic interoceptive deficits across FND and somatic symptom disorders.

\section{Hyperarousal and autonomic hyper-reactivity}

Several studies identified elevated baseline autonomic arousal and HPA-axis activation in people with FND, and others noted similar elevations during experimental emotional processing tasks or in phasic responses to affective stimuli. ${ }^{15} 2733$ 34 41-43

These findings relate to early studies in people with FND, demonstrating more spontaneous electrodermal fluctuations and reduced habituation of SCRs to acoustic probes. ${ }^{68} 69$ Furthermore, cardiac-related autonomic measures show that sympathetic activity is elevated at baseline or preictally in people with FND-seiz, but that it reduces during and/or after seizures,${ }^{70}{ }^{71}$ revealing a possible function of the seizures as a means of reducing heightened physiological/affective arousal, of which some patients are subjectively aware. ${ }^{13} 72$ Elevated HR and reduced vagal tone have also been observed in FND-movt. ${ }^{73}$ Furthermore, Bakvis et al's ${ }^{3474}$ findings concur with other reports of altered HPA-axis functioning in people with FND, ${ }^{6075}$ with two of the studies demonstrating positive associations between HPA-axis markers and adverse life events. ${ }^{6074}$

The observed hyperarousal and increased autonomic reactivity in FND is likely to be related to hyperactivity and diminished habituation of the amygdala observed during affective processing tasks. ${ }^{48-50}$ This profile resembles findings in several other neuropsychiatric disorders and may represent a 'limbic scar' resulting from early or chronic psychosocial stress. ${ }^{7677}$

Similar increased baseline or task-based autonomic/HPA activation have also been observed in other somatic symptom disorders. ${ }^{59} 78$ There is also evidence of reduced pituitary volumes in both individuals with FND and health anxiety. ${ }^{79} 80$ Collectively, these studies draw attention to elevated arousal and autonomic reactivity as an important pathophysiological feature that may be associated with altered social cognition, psychosocial adversity and vulnerability to other somatic symptoms.

\section{Increased motor circuit activity and limbic-motor system connectivity}

Increased activation in motor regions during affective processing has been commonly observed across affective tasks in this review. ${ }^{35} 394047505253$ These findings overlap with previous reports of a range of structural alterations in motor neurocircuits in FND subgroups. Cortical atrophy, for example, has been reported in premotor and motor areas in people with FND-seiz,${ }^{81}$ the former associated with depressive symptoms in that group. In contrast, Aybek et $a l^{82}$ reported cortical thickening of the premotor area in FND-par, and differences in coping through accepting responsibility (ie, an adaptive response) have been associated with individual differences in ventral premotor cortical thickness in mixed FND patients. ${ }^{83}$ SMA volumetric increases were noted in young people with FND, with SMA volumes negatively correlating with reaction times for facial emotion identification. ${ }^{84}$ The SMA is thought to be involved in the selection of action sequences in response to internal cues, whereas the premotor area is involved in action selection in response to external cues. ${ }^{85}$ The cerebellum, which has also been reported to demonstrate group-level volumetric differences in patients with mixed FND $^{57}$ and FND-seiz, ${ }^{81}$ has well-documented roles in coordinating motor-cognitive-affective processing. ${ }^{86}$

Increased limbic-motor circuit connectivity has also been observed during affective processing tasks ${ }^{47-49} 51$ and at rest ${ }^{35}$ in FND samples. It is possible that enhanced limbic-motor coupling might mediate the influence of emotion on voluntary motor control in FND, possibly by contributing to automatic activation or inhibition of motor sequences. ${ }^{87}$ Routes by which this influence might occur potentially involve the insula, ACC or ventral striatum. Additionally, evidence exists for direct projections from the amygdala to voluntary motor cortex in humans and animals ${ }^{88}$ and while these pathways are generally thought to be less dense than other frontolimbic pathways, ${ }^{89}$ it is possible that they could be structurally or functionally stronger in people with FND. 


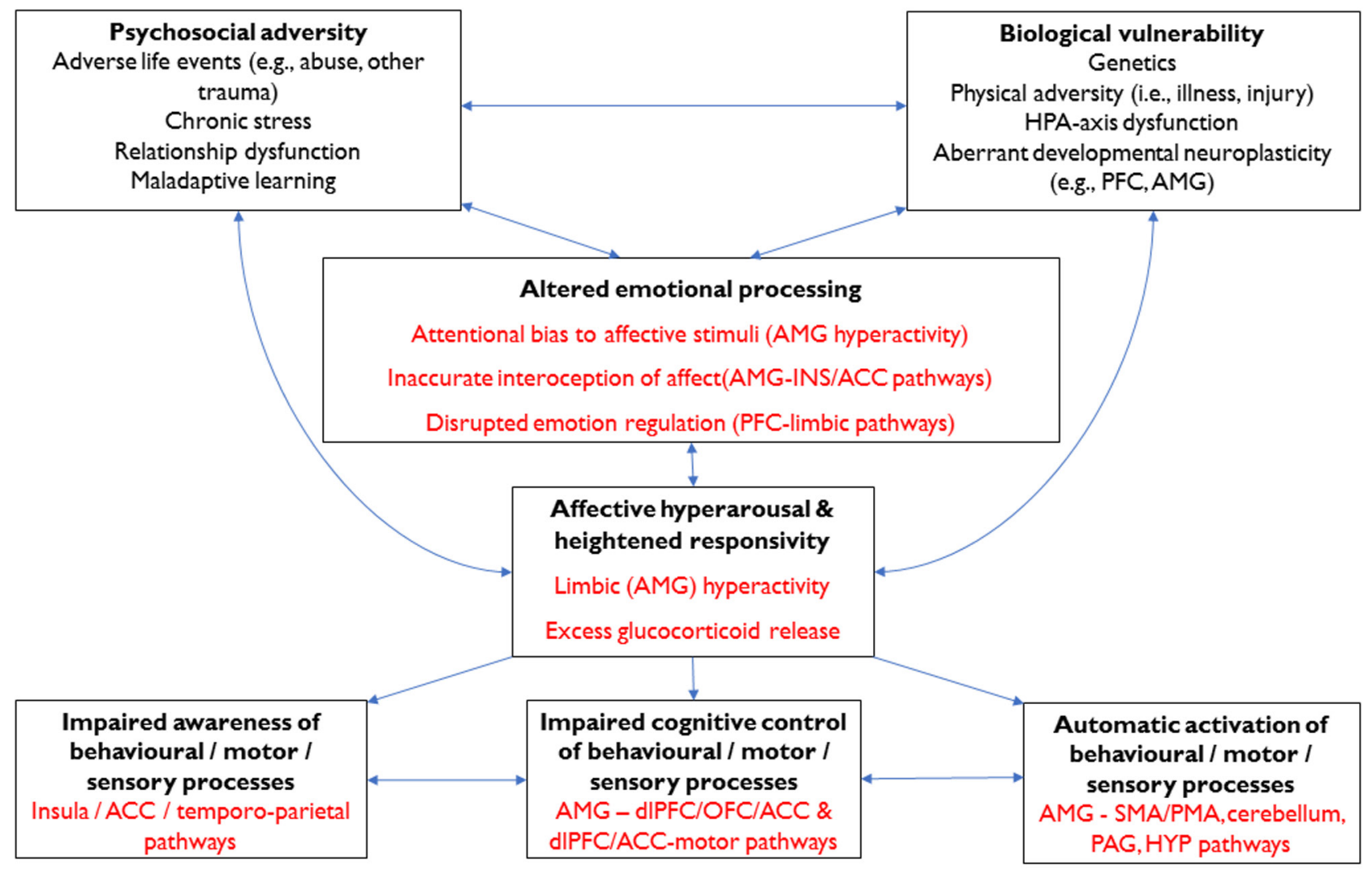

Figure 2 A summary of the possible role of emotional processing in generating (and perpetuating) functional neurological symptoms. Key emotional processing differences include an attentional bias to affective stimuli, inaccurate interoception and suboptimal emotional regulation. Affective hyperarousal and hyper-reactivity are linked to elevated limbic (amygdalar, HPA-axis, PAG) activity which exerts a disruptive influence on neurocircuits crucial for cognitive control, initiation of behavioural/motor responses and awareness. ACC, anterior cingulate gyrus; AMG, amygdala; dIPFC, dorsolateral prefrontal cortex; HPA, hypothalamic-pituitary-adrenal; HYP, hypothalamus; INS, insula; OFC, orbitofrontal cortex; PAG, periaqueductal grey; PFC, prefrontal cortex; PMA, premotor area; SMA, supplementary motor area.

Altered emotion regulation/prefrontal activity

Differences in several prefrontal regions during affective processing tasks have been identified, ${ }^{39} 44475051$ including the dIPFC and cingulate cortex/paracingulate gyrus.

The dIPFC is implicated in the cognitive regulation of emotional states, ${ }^{90} 91$ and the dorsal anterior cingulate cortex (ACC) is known to be important for allocation of cognitive control and regulation of sensorimotor/autonomic affective reactions. ${ }^{92}$ Altered prefrontal engagement during these tasks, therefore, might represent disturbances in the effortful allocation of resources directed towards emotion regulation.

Prefrontal cortex (PFC) regions are closely connected with the amygdala and PAG, so elevated limbic activation could potentially disrupt PFC functioning through reciprocal interactions. Additionally, neuroendocrine stress responses (ie, glucocorticoid and proinflammatory release), mediated by the amygdala and hypothalamus might also adversely impact on the functioning of PFC emotion regulation systems. Nonetheless, more research is needed to understand the specificity of PFC activation profiles in the pathophysiology of FND.

Summary: the possible role of emotional processing in generating and perpetuating FND symptoms

Figure 2 presents an overview of emotional processing alterations in FND and how they may contribute to the generation and perpetuation of FND symptoms. In this model, we propose that a range of vulnerabilities might predispose towards altered emotional processing in people with FND, and these factors are likely to interact and vary between individuals.

Emotional processing alterations in FND include:

- Limbic (amygdalar) hyperactivation, excessive affective (autonomic) arousal and threat-related hypervigilance.

- Impaired interoception of visceromotor emotional responses, possibly promoting reduced emotional awareness and insufficient integration of affective, cognitive and viscerosomatic experiences.

- Suboptimal emotional regulation, possibly including under-regulation and over-regulation at distinct instances in the same individual.

- Disturbances in explicit (subjective) interpretations of affective stimuli (eg, emotion recognition and mentalising).

Altered emotional processing in patients with FND could disrupt awareness and/or higher-order control of a range of other cognitive, behavioural and somatic processes, thereby contributing directly to FND symptom generation, as follows:

- Limbic hyperactivation and autonomic hyperarousal facilitates activation of learnt action sequences (ie, FND-movt; FND-seiz) and/or innate affective responses (eg, autonomic arousal, tonic immobility, psychomotor agitation; FND-seiz; FND-par) via limbic afferents to voluntary motor regions and PAG areas, respectively. 
- Diminished subjective awareness of one or more lowerlevel processes (eg, sensation, behaviour, motor responses) is mediated by disturbed connectivity between limbic regions and those involved with awareness of self, body and behaviour (eg, cingulo-insular, temporoparietal regions).

- Altered ('top-down') control of sensorimotor and affective responses, possibly mediated by altered PFC-amygdala interactions, results in the perceived lack of agency/voluntary control that patients with FND experience.

\section{Methodological considerations}

There are several methodological concerns when interpreting the literature including:

- Sampling

- Most studies included healthy comparison groups only, limiting the extent to which the findings can be viewed as specific to FND.

- FND samples often included comorbid psychiatric disorders, which are common in FND, but few studies adjusted for mood and/or anxiety potentially obscuring result specificity.

- Small sample sizes $(n<20)$ were common, particularly in neuroimaging studies, limiting statistical power and confidence in the results reported.

- Many studies focused on only one subgroup of patients with FND, which does not fully encompass the range of sensorimotor symptoms common in many with FND.

- No studies to date have directly compared FND-subtypes using emotion processing paradigms.

- Psychotropic medications are rarely accounted for, yet such medications (eg, antidepressants, anxiolytics, antiepileptic drugs) likely influence mood, cognition and emotional responsivity.

- Neuropsychological measures of cognitive abilities relevant to task demands have not been routinely used.

- 'Reverse inference ${ }^{93}$ is often used in interpretation of neuroimaging findings as evidence for emotional processing disturbances, sometimes in the absence of subjective, behavioural or psychophysiological alterations.

\section{Future directions}

This review points towards several important avenues for future research, as follows:

1. Replications of existing findings are needed across a broader range of FND symptom types, with additional experimental paradigms and larger samples.

2. Inclusion of both clinical and non-clinical control groups with similar risk factors to FND, such as controls who have experienced trauma and/or mild-moderate anxiety and depression.

3. There is a need for multimodal sampling of emotional processing responses, rather than relying on only behavioural, psychophysiological, neuroendocrine or neuroimaging measures.

4. Relationships between relevant comorbid symptoms (eg, post-traumatic stress disorder, depression, dissociation), psychosocial risk factors (ie, trauma, life events) and emotional processing alterations in FND should be clarified.

5. Future studies should further examine interoceptive awareness in FND samples.

6. Emotional processing should be studied beyond the laboratory setting, to enhance the ecological validity of findings.
7. Additional work is needed to explore the developmental trajectories of emotional processing alterations and associated neurobiological processes in paediatric FND samples.

8. The intersection of emotion processing, motor control, agency perception and somatic sensations should be explored in more detail.

\section{CONCLUSION}

The literature to date indicates heightened preconscious ('bottom-up') processing of emotionally significant stimuli, increased affective arousal, disrupted 'top-down' regulation and altered interoception of bodily emotional responses in people with FND. Furthermore, there is evidence of limbic and motor system hyperactivation, and enhanced interaction of these neurocircuits, during emotional processing in FND samples. We propose that these alterations in emotional processing could contribute directly to the generation of FND symptoms, through enhanced limbic influence on a range of neural circuits involved in awareness and control of multiple lower-level processes, including sensory, motor and behavioural functions. These emotional processing differences might arise from a variety of biological and psychosocial risk factors, including but not limited to aberrant neuroplasticity of corticolimbic circuits associated with early or prolonged psychosocial adversity. This perspective integrates neurobiological and psychosocial processes in a unified model of FND and indicates clear directions for future research.

Contributors TRN and SP formulated the idea and initial plan for the review and DLP contributed to refinement of the structure. SP conducted the literature searches and wrote the first and subsequent drafts. DLP, LHG and TRN contributed to revisions/editing of the manuscript. DLP designed and prepared figure 1, with contributions from SP. SP prepared online supplementary table 1 and figure 2, with contributions on revisions from DLP, LHG and TRN.

Funding DLP was funded by the Sidney R. Baer Jr. Foundation and the National Institute of Mental Health (NIMH) grant K23MH111983-02. SP and TRN were funded by the National Institute of Health Research (NIHR). This manuscript also represents independent research part-funded (LHG) by the NIHR Maudsley Biomedical Research Centre at the South London and Maudsley NHS Foundation Trust and King's College London.

Disclaimer The views expressed are those of the authors and not necessarily those of the NHS, NIHR, Department of Health (UK) or the NIMH (US).

Competing interests DLP has received honorarium from the Movement Disorders Society, American Academy of Neurology and Continuing Medical Education courses at Harvard Medical School.

Patient consent Not required.

Provenance and peer review Not commissioned; externally peer reviewed.

\section{REFERENCES}

1 American Psychiatric Association. Diagnostic and statistical manual of mental disorders. 5th edn. Washington: American Psychiatric Association, 2013.

2 Stone J, Carson A, Duncan R, et al. Who is referred to neurology clinics?--the diagnoses made in 3781 new patients. Clin Neurol Neurosurg 2010;112:747-51.

3 Carson A, Lehn A. Epidemiology. Handb Clin Neurol 2016;139:47-60.

4 World Health Organisation. The ICD-10 classification of mental and behavioural disorders: clinical descriptions and diagnostic guidelines. Geneva: Switzerland: World Health Organisation, 1992.

5 Bodde NM, Brooks JL, Baker GA, et al. Psychogenic non-epileptic seizures--definition, etiology, treatment and prognostic issues: a critical review. Seizure 2009;18:543-53.

6 Reuber M. The etiology of psychogenic non-epileptic seizures: toward a biopsychosocial model. Neurol Clin 2009;27:909-24.

7 Edwards MJ, Adams RA, Brown H, et al. A Bayesian account of 'hysteria'. Brain 2012;135(Pt 11):3495-512.

8 Teodoro T, Edwards MJ, Isaacs JD. A unifying theory for cognitive abnormalities in functional neurological disorders, fibromyalgia and chronic fatigue syndrome: systematic review. J Neurol Neurosurg Psychiatry 2018:jnnp-2017-317823.

9 Van den Bergh O, Witthöft M, Petersen S, et al. Symptoms and the body: taking the inferential leap. Neurosci Biobehav Rev 2017;74-185-203. 
10 Ludwig L, Pasman JA, Nicholson T, et al. Stressful life events and maltreatment in conversion (functional neurological) disorder: systematic review and meta-analysis of case-control studies. Lancet Psychiatry 2018;5:307-20.

11 Stone J, Sharpe M, Binzer M. Motor conversion symptoms and pseudoseizures: a comparison of clinical characteristics. Psychosomatics 2004;45:492-9.

12 Tojek TM, Lumley M, Barkley G, et al. Stress and other psychosocial characteristics of patients with psychogenic nonepileptic seizures. Psychosomatics 2000;41:221-6.

13 Pick S, Mellers JD, Goldstein LH. Emotion and dissociative seizures: a phenomenological analysis of patients' perspectives. Epilepsy Behav 2016;56:5-14.

14 Brown RJ, Reuber M. Psychological and psychiatric aspects of psychogenic nonepileptic seizures (PNES): a systematic review. Clin Psychol Rev 2016;45:157-82.

15 Pick S, Mellers JD, Goldstein LH. Explicit facial emotion processing in patients with dissociative seizures. Psychosom Med 2016;78:874-85.

16 Jalilianhasanpour R, Williams B, Gilman I, et al. Resilience linked to personality dimensions, alexithymia and affective symptoms in motor functional neurological disorders. J Psychosom Res 2018;107:55-61.

17 Kienle J, Rockstroh B, Bohus M, et al. Somatoform dissociation and posttraumatic stress syndrome - two sides of the same medal? A comparison of symptom profiles, trauma history and altered affect regulation between patients with functional neurological symptoms and patients with PTSD. BMC Psychiatry 2017:17:248

18 Myers L, Lancman M, Laban-Grant 0, et al. Psychogenic non-epileptic seizures: predisposing factors to diminished quality of life. Epilepsy Behav 2012;25:358-62.

19 Pick S, Mellers JD, Goldstein LH. Dissociation in patients with dissociative seizures: relationships with trauma and seizure symptoms. Psychol Med 2017;47:1215-29.

20 Novakova B, Howlett S, Baker R, et al. Emotion processing and psychogenic nonepileptic seizures: a cross-sectional comparison of patients and healthy controls. Seizure 2015:29:4-10.

21 Perez DL, Matin N, Barsky A, et al. Cingulo-insular structural alterations associated with psychogenic symptoms, childhood abuse and PTSD in functional neurological disorders. J Neurol Neurosurg Psychiatry 2017;88:491-7.

22 Roelofs K, Keijsers GP, Hoogduin KA, et al. Childhood abuse in patients with conversion disorder. Am J Psychiatry 2002;159:1908-13.

23 Roelofs K, Spinhoven P, Sandijck P, et al. The impact of early trauma and recent life-events on symptom severity in patients with conversion disorder. J Nerv Ment Dis 2005;193:508-14

24 Wolf LD, Hentz JG, Ziemba KS, et al. Quality of life in psychogenic nonepileptic seizures and epilepsy: the role of somatization and alexithymia. Epilepsy Behav 2015:43:81-8.

25 Selkirk M, Duncan R, Oto M, et al. Clinical differences between patients with nonepileptic seizures who report antecedent sexual abuse and those who do not. Epilepsia 2008;49:1446-50.

26 Goldstein LH, Chalder T, Chigwedere C, et al. Cognitive-behavioral therapy for psychogenic nonepileptic seizures: a pilot RCT. Neurology 2010;74:1986-94.

27 Bakvis P, Roelofs K, Kuyk J, et al. Trauma, stress, and preconscious threat processing in patients with psychogenic nonepileptic seizures. Epilepsia 2009;50:1001-11.

28 Bakvis P, Spinhoven P, Roelofs K. Basal cortisol is positively correlated to threat vigilance in patients with psychogenic nonepileptic seizures. Epilepsy Behav 2009;16:558-60.

29 Pick S, Mellers JDC, Goldstein LH. Implicit attentional bias for facial emotion in dissociative seizures: Additional evidence. Epilepsy Behav 2018;80:296-302.

30 Bakvis P, Spinhoven P, Putman P, et al. The effect of stress induction on working memory in patients with psychogenic nonepileptic seizures. Epilepsy Behav 2010:19:448-54.

31 Gul A, Ahmad H. Cognitive deficits and emotion regulation strategies in patients with psychogenic nonepileptic seizures: a task-switching study. Epilepsy Behav 2014:32:108-13.

32 Kozlowska K, Brown KJ, Palmer DM, et al. Specific biases for identifying facial expression of emotion in children and adolescents with conversion disorders. Psychosom Med 2013;75:272-80.

33 Kozlowska K, Palmer DM, Brown KJ, et al. Reduction of autonomic regulation in children and adolescents with conversion disorders. Psychosom Med 2015;77:356-70.

34 Bakvis $\mathrm{P}$, Spinhoven $\mathrm{P}$, Zitman FG, et al. Automatic avoidance tendencies in patients with psychogenic non-epileptic seizures. Seizure 2011;20:628-34.

35 Szaflarski JP, Allendorfer JB, Nenert R, et al. Facial emotion processing in patients with seizure disorders. Epilepsy Behav 2018;79:193-204.

36 Schönenberg $M$, Jusyte $A$, Höhnle $N$, et al. Theory of mind abilities in patients with psychogenic nonepileptic seizures. Epilepsy Behav 2015;53:20-4.

37 Stonnington CM, Locke DE, Hsu CH, et al. Somatization is associated with deficits in affective Theory of Mind. J Psychosom Res 2013;74:479-85.

38 Lang PJ, Bradley MM, Cuthbert BN. International affective picture system (IAPS): instruction manual and affective ratings. University of Florida, 2005.

39 Blakemore RL, Sinanaj I, Galli S, et al. Aversive stimuli exacerbate defensive motor behaviour in motor conversion disorder. Neuropsychologia 2016;93-229-41.

40 Fiess J, Rockstroh B, Schmidt R, et al. Emotion regulation and functional neurological symptoms: Does emotion processing convert into sensorimotor activity? J Psychosom Res 2015;79:477-83.
41 Pick S, Mellers JDC, Goldstein LH. Autonomic and subjective responsivity to emotional images in people with dissociative seizures. J Neuropsychol 2018;12:341-55.

42 Roberts NA, Burleson MH, Weber DJ, et al. Emotion in psychogenic nonepileptic seizures: responses to affective pictures. Epilepsy Behav 2012;24:107-15.

43 Seignourel PJ, Miller K, Kellison I, et al. Abnormal affective startle modulation in individuals with psychogenic [corrected] movement disorder. Mov Disord 2007;22:1265-71.

44 Morris LS, BT B, Baek K, et al. Disrupted avoidance learning in functional neurological disorder. Neurolmage Clin 2017;16:286-94.

45 Kranick SM, Moore JW, Yusuf N, et al. Action-effect binding is decreased in motor conversion disorder: implications for sense of agency. Mov Disord 2013;28:1110-6.

46 Brown LB, Nicholson TR, Aybek S, et al. Neuropsychological function and memory suppression in conversion disorder. J Neuropsychol 2014;8:171-85.

47 Aybek S, Nicholson TR, Zelaya F, et al. Neural correlates of recall of life events in conversion disorder. JAMA Psychiatry 2014;71:52-60.

48 Voon V, Brezing C, Gallea C, et al. Emotional stimuli and motor conversion disorder. Brain 2010;133(Pt 5):1526-36.

49 Hassa T, Sebastian A, Liepert J, et al. Symptom-specific amygdala hyperactivity modulates motor control network in conversion disorder. Neuroimage Clin 2017; 15:143-50.

50 Aybek S, Nicholson TR, O'Daly 0, et al. Emotion-motion interactions in conversion disorder: an FMRI study. PLoS One 2015;10:e0123273.

51 Espay AJ, Maloney T, Vannest J, et al. Impaired emotion processing in functional (psychogenic) tremor: a functional magnetic resonance imaging study. Neuroimage Clin 2018;17:179-87

52 Espay AJ, Maloney T, Vannest J, et al. Dysfunction in emotion processing underlies functional (psychogenic) dystonia. Mov Disord 2018;33:136-45.

53 Fiess J, Rockstroh B, Schmidt R, et al. Functional neurological symptoms modulate processing of emotionally salient stimuli. J Psychosom Res 2016;91:61-7.

54 Tamietto M, de Gelder B. Neural bases of the non-conscious perception of emotional signals. Nat Rev Neurosci 2010;11:697-709.

55 Atmaca M, Aydin A, Tezcan E, et al. Volumetric investigation of brain regions in patients with conversion disorder. Prog Neuropsychopharmacol Biol Psychiatry 2006;30:708-13

56 Nicholson TR, Aybek S, Kempton MJ, et al. A structural MRI study of motor conversion disorder: evidence of reduction in thalamic volume. J Neurol Neurosurg Psychiatry 2014:85:227-9.

57 Perez DL, Williams B, Matin N, et al. Corticolimbic structural alterations linked to health status and trait anxiety in functional neurological disorder. J Neurol Neurosurg Psychiatry 2017;88:1052-9

58 Pedrosa Gil F, Ridout N, Kessler H, et al. Facial emotion recognition and alexithymia in adults with somatoform disorders. Depress Anxiety 2009;26:E26-E33.

59 Pollatos 0 , Herbert BM, Wankner S, et al. Autonomic imbalance is associated with reduced facial recognition in somatoform disorders. J Psychosom Res 2011;71:232-9.

60 Apazoglou K, Mazzola V, Wegrzyk J, et al. Biological and perceived stress in moto functional neurological disorders. Psychoneuroendocrinology 2017;85:142-50.

61 Ricciardi L, Demartini B, Crucianelli L, et al. Interoceptive awareness in patients with functional neurological symptoms. Biol Psychol 2016;113:68-74.

62 Stone J, Zeman A, Simonotto E, et al. FMRI in patients with motor conversion symptoms and controls with simulated weakness. Psychosom Med 2007:69:961-9.

63 Voon V, Brezing C, Gallea C, et al. Aberrant supplementary motor complex and limbic activity during motor preparation in motor conversion disorder. Mov Disord 2011;26:2396-403.

64 Ding JR, An D, Liao W, et al. Altered functional and structural connectivity networks in psychogenic non-epileptic seizures. PLoS One 2013;8:e63850.

65 van der Kruijs SJ, Jagannathan SR, Bodde NM, et al. Resting-state networks and dissociation in psychogenic non-epileptic seizures. J Psychiatr Res 2014;54:126-33.

66 Bogaerts K, Van Eylen L, Li W, et al. Distorted symptom perception in patients with medically unexplained symptoms. J Abnorm Psychol 2010;119:226-34.

67 Schaefer M, Egloff B, Gerlach AL, et al. Improving heartbeat perception in patients with medically unexplained symptoms reduces symptom distress. Biol Psychol 2014;101:69-76.

68 Horvath T, Friedman J, Meares R. Attention in hysteria: a study of Janet's hypothesis by means of habituation and arousal measures. Am J Psychiatry 1980;137:217-20.

69 Lader M, Sartorius N. Anxiety in patients with hysterical conversion symptoms. J Neurol Neurosurg Psychiatry 1968:31:490-5.

70 Ponnusamy A, Marques JL, Reuber M. Heart rate variability measures as biomarkers in patients with psychogenic nonepileptic seizures: potential and limitations. Epilepsy Behav 2011;22:685-91.

71 Reinsberger C, Perez DL, Murphy MM, et al. Pre- and postictal, not ictal, heart rate distinguishes complex partial and psychogenic nonepileptic seizures. Epilepsy Behav 2012;23:68-70.

72 Stone J, Carson AJ. The unbearable lightheadedness of seizing: wilful submission to dissociative (non-epileptic) seizures. J Neurol Neurosurg Psychiatry 2013:84:822-4.

73 Maurer CW, Liu VD, LaFaver K, et al. Impaired resting vagal tone in patients with functional movement disorders. Parkinsonism Relat Disord 2016;30:18-22.

74 Bakvis P, Spinhoven P, Giltay EJ, et al. Basal hypercortisolism and trauma in patients with psychogenic nonepileptic seizures. Epilepsia 2010;51:752-9. 
75 Tunca Z, Fidaner $\mathrm{H}$, Cimilli C, et al. Is conversion disorder biologically related with depression?: a DST study. Biol Psychiatry 1996;39:216-9.

76 Dannlowski U, Stuhrmann A, Beutelmann V, et al. Limbic scars: long-term consequences of childhood maltreatment revealed by functional and structural magnetic resonance imaging. Biol Psychiatry 2012;71:286-93.

77 Teicher MH, Samson JA. Annual Research Review: Enduring neurobiological effects of childhood abuse and neglect. J Child Psychol Psychiatry 2016;57:241-66.

78 Rief W, Shaw R, Fichter MM. Elevated levels of psychophysiological arousal and cortisol in patients with somatization syndrome. Psychosom Med 1998;60:198-203.

79 Atmaca M, Yildirim H, Sec S, et al. Pituitary volumes in hypochondriac patients. Prog Neuropsychopharmacol Biol Psychiatry 2010;34:344-7.

80 Atmaca M, Baykara S, Mermi 0, et al. Pituitary volumes are changed in patients with conversion disorder. Brain Imaging Behav 2016;10:92-5.

81 Labate A, Cerasa A, Mula M, et al. Neuroanatomic correlates of psychogenic nonepileptic seizures: a cortical thickness and VBM study. Epilepsia 2012;53:377-85.

82 Aybek S, Nicholson TR, Draganski B, et al. Grey matter changes in motor conversion disorder. J Neurol Neurosurg Psychiatry 2014:85:236-8.

83 Williams B, Jalilianhasanpour R, Matin N, et al. Individual differences in corticolimbic structural profiles linked to insecure attachment and coping styles in motor functional neurological disorders. J Psychiatr Res 2018;102:230-7.
84 Kozlowska K, Griffiths KR, Foster SL, et al. Grey matter abnormalities in children and adolescents with functional neurological symptom disorder. Neuroimage Clin 2017; 15:306-14.

85 Passingham RE. The frontal lobes and voluntary action. Oxford University Press: Oxford, 1993.

86 Timmann D, Drepper J, Frings $\mathrm{M}$, et al. The human cerebellum contributes to motor, emotional and cognitive associative learning. A review. Cortex 2010;46:845-57.

87 Vuilleumier P. Brain circuits implicated in psychogenic paralysis in conversion disorders and hypnosis. Neurophysiol Clin 2014;44:323-37.

88 Grèzes J, Valabrègue R, Gholipour B, et al. A direct amygdala-motor pathway for emotional displays to influence action: A diffusion tensor imaging study. Hum Brain Mapp 2014;35:5974-83.

89 Freese JL, Amaral DG. Neuroanatomy of the primate amygdala. In: . The Human Amygdala. Whalen PJ, Phelps EA. New York: Guildford Press, 2009: 3-42.

90 Dixon ML, Thiruchselvam $\mathrm{R}$, Todd $\mathrm{R}$, et al. Emotion and the prefrontal cortex: an integrative review. Psychol Bull 2017;143:1033-81.

91 Etkin A, Büchel C, Gross JJ. The neural bases of emotion regulation. Nat Rev Neurosci 2015;16:693-700

92 Shackman AJ, Salomons TV, Slagter HA, et al. The integration of negative affect, pain and cognitive control in the cingulate cortex. Nat Rev Neurosci 2011;12:154-67.

93 Poldrack RA. Can cognitive processes be inferred from neuroimaging data? Trends Cogn Sci 2006;10:59-63. 